\title{
Association between urinary metabolic profile and the intestinal effects of cocoa in rats
}

\author{
Malen Massot-Cladera ${ }^{1,2} \dagger$, Jordi Mayneris-Perxachs ${ }^{3} \dagger$, Adele Costabile ${ }^{4}$, Jonathan R. Swann ${ }^{3}$, \\ Àngels Franch ${ }^{1,2}$, Francisco J. Pérez-Cano ${ }^{1,2 *}$ and Margarida Castell ${ }^{1,2}$ \\ ${ }^{1}$ Department of Biochemistry and Physiology, Faculty of Pharmacy and Food Sciences, University of Barcelona, \\ Av. Joan XXIII, 27-31, Building B, 3rd Floor, Barcelona 08028, Spain \\ ${ }^{2}$ Nutrition and Food Safety Research Institute (IrNSA), University of Barcelona, Prat de la Riba, 171, Santa Coloma de \\ Gramenet 08921, Spain \\ ${ }^{3}$ Division of Computational and Systems Medicine, Imperial College London, London SW7 2AZ, UK \\ ${ }^{4}$ Health Sciences Research Centre, Life Science Department, Whitelands College, University of Roehampton, Holybourne \\ Avenue, London SW15 4JD, UK \\ (Submitted 16 September 2016 - Final revision received 22 January 2017 - Accepted 7 February 2017- First published online 27 March 2017)
}

\begin{abstract}
The aim of this study was to elucidate the relationship between the urinary metabolic fingerprint and the effects of cocoa and cocoa fibre on body weight, hormone metabolism, intestinal immunity and microbiota composition. To this effect, Wistar rats were fed, for 3 weeks, a diet containing $10 \%$ cocoa (C10) or two other diets with same the proportion of fibres: one based on cocoa fibre (CF) and another containing inulin as a reference (REF) diet. The rats' $24 \mathrm{~h}$ urine samples were analysed by an untargeted ${ }^{1} \mathrm{H}$ NMR spectroscopy-based metabonomic approach. Concentrations of faecal IgA and plasma metabolic hormones were also quantified. The C10 diet decreased the intestinal IgA, plasma glucagon-like peptide-1 and glucagon concentrations and increased ghrelin levels compared with those in the REF group. Clear differences were observed between the metabolic profiles from the $\mathrm{C} 10$ group and those from the CF group. Urine metabolites derived from cocoa correlated with the cocoa effects on body weight, immunity and the gut microbiota. Overall, cocoa intake alters the host and bacterial metabolism concerning energy and amino acid pathways, leading to a metabolic signature that can be used as a marker for consumption. This metabolic profile correlates with body weight, metabolic hormones, intestinal immunity and microbiota composition.
\end{abstract}

Key words: Cocoa: Hormones: IgA: Metabonomics: Microbiota

Cocoa is considered a great source of bioactive compounds such as polyphenols and dietary fibre (DF), which have been attributed consistent positive health effects ${ }^{(1-5)}$. Cocoa contains monomeric flavonoids, such as the flavanols (-)-catechin, $(+)$-catechin and (-)-epicatechin, and mainly its oligomers and polymers known as procyanidins. These polymeric compounds are able to pass intact through the small intestine and reach the colon $^{(6)}$, where they are metabolised by the intestinal microbiota. This conversion is crucial for their absorption and also for the generation of new compounds with greater bioactivity than their precursors ${ }^{(6-8)}$. Thus, there is a growing body of evidence on the reciprocal relationship between bacteria and polyphenols that may help understand the documented benefits of polyphenols consumption $^{(9)}$. In fact, it has been extensively reported that both dietary polyphenols, including those from cocoa, and the corresponding microbially-derived phenolic metabolites modulate the gut microbiota composition in in vitro, in vivo and clinical studies ${ }^{(8,10-13)}$.

A similar ability to modulate the gut microbiota composition has been attributed to DF, which has been described to modulate SCFA production ${ }^{(14-18)}$. Recently, cocoa fibre-enriched intake in rats has been associated with the modulation of gut microbiota and SCFA production ${ }^{(17)}$. The DF fraction in cocoa is mainly rich in cellulose, followed by highly fermented pectic substances and hemicellulose, which is less fermentable than the former ${ }^{(19)}$. After microbial transformation, the cocoa metabolites either from flavonoids or cocoa fibre (i.e. SCFA) are absorbed into the bloodstream ${ }^{(20,21)}$. The microbial metabolites

Abbreviations: 4-HPA, 4-hydroxypropionic acid; 3-IS, 3-indoxyl-sulfate; C10, $10 \%$ cocoa diet; CF, cocoa fibre diet; IAA, indole-3-acetic acid; NMN, nicotine mononucleotide; NMND, $N$-methylnicotinamide; OPLS-DA, orthogonal projection to latent structures-discriminant analysis; PAG, phenylacetylglycine; REF, reference diet.

* Corresponding author: F. J. Pérez-Cano, fax +3493403 5901, email franciscoperez@ub.edu

$\dagger$ These authors contributed equally to this work. 
from flavanols are mainly metabolised by liver phase-II enzymes to conjugated derivatives that are subsequently eliminated in urine ${ }^{(7)}$.

Previous preclinical studies carried out in our laboratory have evidenced that cocoa, cocoa flavanols and cocoa fibre modify some aspects of the intestinal and systemic immune response $\mathrm{e}^{(2,13,17,22)}$. On the other hand, a diet based on whole cocoa, but not based exclusively on its flavonoids or fibre, is able to reduce body weight gain ${ }^{(13,17,23)}$. Given that this effect on weight is not associated with a lower chow intake, it is necessary to further understand the mechanism involved in such an effect. In this sense, an untargeted metabonomic approach is particularly useful, as it aims to determine the broadest range of metabolites present in a biological sample without a priori information. Thus, due to its exploratory nature, this hypothesisgenerating approach provides an unbiased screening of the metabolites and can detect alterations in biological pathways to provide an insight into their molecular mechanisms. Therefore, the objective of this study was to correlate the urinary metabolic variation associated with cocoa intake identified by ${ }^{1} \mathrm{H}$ NMR spectroscopy with the effects of cocoa on body weight, hormone metabolism, intestinal immunity and microbiota composition. Moreover, very limited data are available on the metabolic profile after cocoa fibre intake and even less on the contribution of cocoa fibre to the above-described effects ascribed to cocoa; thus, it was also included as an aim of this study.

\section{Methods}

\section{Animals and diets}

Female Wistar rats (3-week-old) were obtained from Janvier (Saint-Berthevin, France) and housed in pairs under conditions of controlled temperature and humidity in a $12 \mathrm{~h}$ light- $12 \mathrm{~h}$ dark cycle. The rats were randomly distributed into three dietary groups: $10 \%$ cocoa (C10), cocoa fibre (CF) and reference (REF) groups ( $n 10$ in each). The $\mathrm{C} 10$ group received chow containing $10 \%$ cocoa that provided a final diet concentration of $0.4 \%$ polyphenols, $0.85 \%$ soluble fibre and $2.55 \%$ insoluble fibre; the $\mathrm{CF}$ group received a diet with the same cocoa soluble and insoluble fibre proportions as the $\mathrm{C} 10$ group but with a very low amount of polyphenols ( $<0.02 \%)$; and the REF group received the same amount of fibre as the C10 group, with the soluble portion $(0.85 \%)$ as inulin in order to distinguish the particular effect of cocoa fibre, as has been previously reported ${ }^{(24)}$. Natural Forastero cocoa and cocoa fibre powders (provided by Idilia Foods S.L., formerly Nutrexpa S.L.) with 4.02 and $0.35 \%$ of polyphenols, respectively, were used to elaborate the $\mathrm{C} 10$ and $\mathrm{CF}$ diets. Inulin from chicory roots (Fibruline ${ }^{\circledR}$ Instant; InnovaFood 2005, S.L.) was used as a reference soluble fibre. The three experimental diets were elaborated on the basis of the AIN-93M formula by subtracting the amount of carbohydrates, proteins, lipids and insoluble fibre provided by the corresponding supplement. The three resulting diets were isoenergetic and had the same proportion of macronutrients (carbohydrates, proteins and lipids) and fibre as has been previously stated ${ }^{(24)}$. Animals were given free access to water and food. The diets lasted for 3 weeks.
Body weight and food intake were monitored throughout the study. Experiments were performed according to the Guide for the Care and Use of Laboratory Animals, and experimental procedures were approved by the Ethical Committee for Animal Experimentation of the University of Barcelona (ref. 358/12).

\section{Sample collection and processing}

The $24 \mathrm{~h}$ urine samples were collected, by means of metabolic cages, at $15 \mathrm{~d}$ after beginning the nutritional intervention. Centrifuged urine samples were kept at $-80^{\circ} \mathrm{C}$ until analysis. Moreover, blood samples were collected after 3 weeks and plasma was kept at $-80^{\circ} \mathrm{C}$ before metabolic hormones determination. Faecal samples were also collected at the 3rd week of diet, and the homogenates were obtained as previously described $^{(17)}$ and frozen at $-20^{\circ} \mathrm{C}$ until analysis.

\section{Sample preparation for ${ }^{1} \mathrm{H} N \mathrm{~N} R$ analysis}

Urine samples were defrosted and prepared for ${ }^{1} \mathrm{H}$ NMR spectroscopy by combining $400 \mu \mathrm{l}$ of sample with $200 \mu \mathrm{l}$ of PBS ( $\mathrm{pH} 7 \cdot 4 ; 100 \% \mathrm{D}_{2} \mathrm{O}$ ) containing $1 \mathrm{~mm}$ of 3-trimethylsilyl$1-\left[2,2,3,3-{ }^{2} \mathrm{H}_{4}\right]$ propionate (TSP) as an external standard and $2 \mathrm{~mm}$ sodium azide as a bacteriocide. Samples were vortexed and particles were removed by centrifugation (13000 $\boldsymbol{g}$ for $10 \mathrm{~min}$ ) before transferring $550 \mu \mathrm{l}$ into a $5 \mathrm{~mm}$ NMR tube. Standard one-dimensional ${ }^{1} \mathrm{H}$ NMR spectra of the urine samples were acquired on a $500 \mathrm{MHz}$ Bruker NMR spectrometer using a standard nuclear Overhauser effect spectroscopy (NOESY) experiment incorporating a presaturation pulse to attenuate the water signal. This experiment consisted of recycle delay (RD)$90^{\circ}-t_{1}-90^{\circ}$-mixing time $\left(t_{\mathrm{m}}\right)-90^{\circ}$-acquire free induction decay. The water signal was suppressed by irradiation during the RD of $2 \mathrm{~s}$, with a $t_{\mathrm{m}}$ of $10 \mu \mathrm{s}$. The acquisition time was set to $2.91 \mathrm{~s}$ and the $90^{\circ}$ pulse length was $15.87 \mu$ s. For each sample, eight dummy scans were followed by 128 scans and collected in $64 \mathrm{~K}$ data points using a spectral width of 16 parts per million (ppm). Before data analysis, NMR spectra were phased, corrected for baseline distortions and calibrated using the reference standard TSP. ${ }^{1} \mathrm{H}$ NMR spectra $(\delta \quad 0 \cdot 2-10 \cdot 0)$ were digitised into consecutive integrated spectral regions (approximately 20000) of equal width $(0.00055 \mathrm{ppm})$ using MATLAB (MathWorks). The regions containing signals from urea $(\delta$ 5.5-6.0) and residual water $(\delta 4 \cdot 7-5 \cdot 2)$ were removed to minimise baseline effects arising from imperfect water suppression. Chemical shift variation was minimised across the data set by applying a recursive segment-wise peak alignment algorithm to each spectrum. Each spectrum was normalised to unit area to account for variation in sample concentration.

\section{Quantification of metabolic hormones in plasma}

Plasma concentrations of ghrelin, glucagon, glucagon-like peptide-1 (GLP-1) and leptin were determined in plasma using the Bio-Plex Pro ${ }^{\mathrm{TM}}$ Diabetes Assay (Bio-Rad) according to the manufacturer's instructions. Analysis was carried out with the Bio-Plex ${ }^{\circledR}$ MAGPIX ${ }^{\mathrm{TM}}$ Multiplex Reader and Bio-Plex Data Pro $^{\mathrm{TM}}$ software (Bio-Rad) as in previous studies ${ }^{(25)}$. 
The limits of quantification can be found in the online Supplementary Material.

\section{Faecal IgA quantification}

The concentration of IgA in faeces was quantified by ELISA following the manufacturer's instructions (Bethyl Laboratories, Inc.). Absorbance was measured in a microplate photometer (LabSystems Multiskan) and data were interpolated using ASCENT version 2.6 software (Thermo Fisher Scientific) into standard curves.

\section{Statistical analysis}

The number of animals used in each group for detecting a statistically significant difference among groups assuming that there is no dropout rate and type I error of 0.05 (two-sided) was calculated by the Appraising Project Office's program from the Universidad Miguel Hernández de Elche (Alicante) taking into account both the metabolic hormones, particularly the leptin and ghrelin concentrations, and the faecal IgA. Moreover, we have adjusted the sample size to the minimum needed by following the University Ethical Committee guidelines and trying to apply the three R's rule for experimenting in animals.

Statistical analysis for body weight, chow intake, faecal IgA and metabolic hormones was performed using the software package IBM SPSS Statistics 22.0 (SPSS Inc.). Levene's and Kolmogorov-Smirnov tests were applied to assess variance equality and normal distribution, respectively. Conventional one-way ANOVA was performed when normal distribution and equality of variance existed. Tukey's test was applied when specific cocoa intake had a significant effect on the dependent variable. Non-parametric Mann-Whitney $U$ and Wilcoxon's tests were used in order to assess significance for independent and related samples, respectively. Significant differences were established at $P<0.05$

Multivariate modelling was performed in MATLAB using in-house scripts. This included principal components analysis using pareto-scaled data and orthogonal projection to latent structures-discriminant analysis (OPLS-DA) using a unit variance scaling approach. Pairwise OPLS-DA models were constructed to aid model interpretation and identify discriminatory metabolites between the study groups. Here, ${ }^{1} \mathrm{H}$ NMR spectroscopic profiles served as the descriptor matrix $(X)$ and the experimental groups (REF, C10, CF) were used pairwise as the response variable $(Y)$. Orthogonal signal correction filters were used to remove variation in the descriptor matrix unrelated to the response variable to assist model interpretation. Loading coefficient plots were generated by back-scaling transformation where covariance is plotted between the $Y$-response matrix and the signal intensity of the metabolites in the NMR data $(X)$. These plots are coloured based on the correlation coefficient $\left(r^{2}\right)$ between each metabolite and the $Y$-response variable, with red indicating strong significance and blue indicating weak significance. The predictive performance $\left(Q^{2} \mathrm{Y}\right)$ of the model was calculated using a 7-fold cross-validation approach and model validity was established by permutation testing (1000 permutations).
Clustering analysis. Unsupervised hierarchical clustering analysis (HCA) was performed to identify general patterns of metabonomic variation between samples. To do so, we used the normalised levels of metabolites identified to contribute to class separation through the OPLS-DA models. For comparative analysis across different metabolites, data were standardised as $z$-scores across samples for each metabolite before clustering, so that the mean was 0 and the SD was 1 . This standardised matrix was subsequently used in unsupervised HCA for samples and metabolites using Euclidean distance and average linkage, by means of the pdist and linkage functions in the MATLAB bioinformatics toolbox. Heatmaps and dendrograms following HCA were generated with MATLAB imagesc and dendrogram functions, respectively. In the heatmaps, a red-blue colour scale is used such that shades of red and blue represent higher and lower values, respectively, compared with the mean. Different diet groups are colour-coded and shown under the dendrogram for each sample.

Correlation analysis. To explore the functional correlation between the changes on body weight, metabolism and intestinal immunity, metabonome perturbations and gut microbiome evaluated in a previous study by fluorescence in situ hybridisation coupled with flow cytometry, Spearman's correlation analyses were performed on all the samples grouped together. The Benjamini-Hochberg method was used to adjust $P$ values for multiple testing considering a $5 \%$ false discovery rate.

\section{Results}

\section{Body weight and food intake}

Body weight and food intake were monitored weekly throughout the study (Fig. 1). Initially, no differences in body weight were observed between groups (REF: mean 43.7 (sem 1.1) g; C10: mean 45.4 (SEM 1.4) g; CF: mean 44.8 (sEm 1.1) g). However, from day 7 , a lower body weight gain was observed in C10 animals compared with that in the other groups $(P<0.05)$ (Fig. 1(a)). This effect was not related to lower food intake, which was similar throughout the study among all experimental groups (Fig. 1(b)), being approximately $15 \mathrm{~g}$ of chow per $100 \mathrm{~g}$ of animal the 1st week of diet and approximately $12 \mathrm{~g}$ of chow per $100 \mathrm{~g}$ after the 3 weeks of intervention. No changes in body weight gain were found as a result of CF diet intake.

\section{Metabolic hormones}

The metabolic hormones quantified in plasma after the 3-week dietary intervention for all groups are summarised in Table 1. Both the $\mathrm{C} 10$ and $\mathrm{CF}$ diets increased the concentration of ghrelin compared with the REF group $(P<0 \cdot 05)$. This increase was higher in the $\mathrm{C} 10$ group compared with that in the $\mathrm{CF}$ group $(P<0 \cdot 05)$. Both diets also resulted in a lower plasma GLP-1 concentration in comparison with that in the REF group $(P<0.05)$. Moreover, the $\mathrm{C} 10$ diet reduced the glucagon concentration compared with the REF and CF diets $(P<0.05)$. 


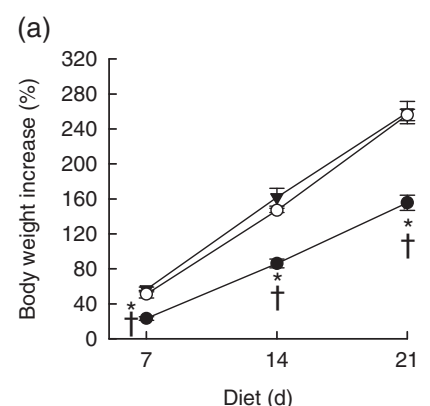

(b)

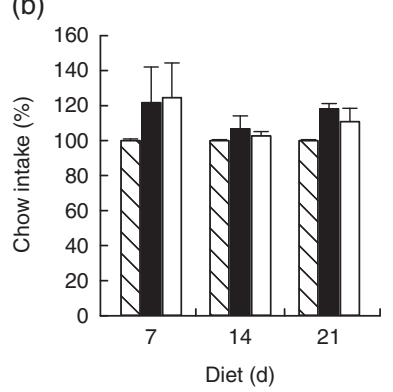

Fig. 1. Body weight increase (\%) compared with baseline (a) and chow intake (\%) compared with the reference (REF) diet which represents $100 \%$ (b) monitored throughout the nutritional intervention. a: $₫$, REF; $\square, 10 \%$ cocoa (C10); $\square$, cocoa fibre (CF); b: $\longrightarrow$, REF; $-\longrightarrow, C 10 ;-O-$, CF. Values are means ( $n$ 10) with their standard errors. ${ }^{*} P<0.05 \mathrm{v}$. REF diet; $† P<0.05 \mathrm{v}$. CF diet.

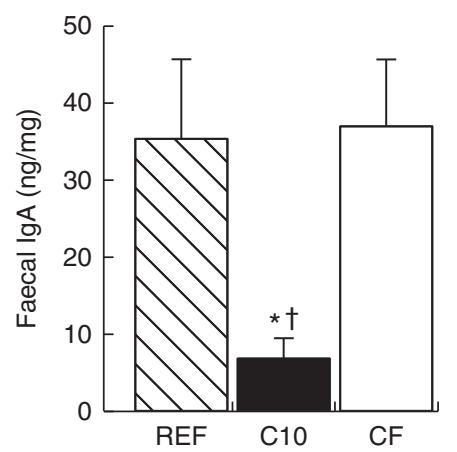

Fig. 2. Faecal $\lg \mathrm{A}$ concentration determined after 3 weeks of nutritional intervention. REF, Reference; C10, 10\% cocoa; CF, cocoa fibre. Values are means ( $n$ 9-10), with their standard errors represented by vertical bars. ${ }^{*} P<0.05$ v. REF diet; $\dagger P<0.05$ v. CF diet.

Table 1. Metabolic hormones in plasma after 3 weeks of nutritional intervention (Mean values with their standard errors; $n 7$ )

\begin{tabular}{|c|c|c|c|c|c|c|}
\hline & \multicolumn{2}{|c|}{ REF } & \multicolumn{2}{|c|}{$\mathrm{C} 10$} & \multicolumn{2}{|c|}{$\mathrm{CF}$} \\
\hline & Mean & SEM & Mean & SEM & Mean & SEM \\
\hline Ghrelin (ng/ml) & 30.57 & 3.9 & $98.63^{\star} \ddagger$ & $18 \cdot 7$ & $43.59^{*}$ & 4.3 \\
\hline GLP-1 (pg/ml) & 29.4 & $16 \cdot 4$ & $7 \cdot 4^{*}$ & $3 \cdot 1$ & $4 \cdot 2^{*}$ & 0.2 \\
\hline Glucagon (pg/ml) & $180 \cdot 1$ & $25 \cdot 3$ & $100 \cdot 4^{\star} \ddagger$ & $3 \cdot 1$ & $166 \cdot 2$ & $26 \cdot 9$ \\
\hline Leptin (pg/ml) & $647 \cdot 7$ & 135.5 & 335.4 & $127 \cdot 5$ & $968 \cdot 7^{*} \dagger$ & $177 \cdot 6$ \\
\hline
\end{tabular}

REF, reference diet; C10, $10 \%$ cocoa diet; CF, cocoa fibre diet; GLP-1, glucagon-like peptide-1.

* $P<0.05$ v. REF diet, $\dagger P<0.05$ v. C10 diet, $\ddagger P<0.05$ v. CF diet.

Leptin concentration was not affected after the C10 diet, but it was up-regulated as a result of the $\mathrm{CF}$ diet intake compared with that in the rest of the groups $(P<0 \cdot 05)$ (Table 1$)$.

\section{Faecal IgA}

The C10 diet intake resulted in a decrease in the secretory IgA concentration compared with that in the other groups $(P<0.05)$ (Fig. 2). The CF diet did not produce any change in the secreted IgA concentration, which was similar to that quantified in the REF group.

\section{Urinary metabolic profile}

An OPLS-DA model with strong predictive ability $\left(Q^{2} \mathrm{Y}=0.93\right.$; $P=0.001)$ was returned comparing the metabolic profiles from rats receiving the $\mathrm{C} 10$ diet and the REF diet (Fig. 3(a) and 4). Rats fed the $\mathrm{C} 10$ diet excreted cocoa-derived metabolites such as $N$-methylnicotinic acid (NMNA) and nicotine mononucleotide (NMN), as well as methylxanthine metabolites, that is, theobromine, xanthine, 1-methylxanthine (1-MX), 3-methylxanthine (3-MX), 7-methylxanthine (7-MX), imidazole, dimethyluric acid (DMU). In addition, urine samples from the C10 group contained microbial-derived metabolites such as hippurate and 2-hydroxyisobutyrate (2-HIB), and 4-hydroxypropionic acid (4-HPA), phenylacetylglycine (PAG) and indole-3-acetic acid (IAA), which could be derived from the cocoa polyphenols (epicatechin and procyanidins), amino acids (tyrosine, phenylalanine and trypthophan) or monoamines (tyramine, tryptamine and 2-phenylethylamine) contained in cocoa. Moreover, rats receiving the $\mathrm{C} 10$ diet also excreted greater amounts of taurine compared with those receiving the REF diet. Conversely, animals receiving the C10 diet excreted lower amounts of metabolites related to energy metabolism (acetone, citrate, 2-oxoglutarate (2-OG), $\mathrm{N}$-methylnicotinamide (NMND)), choline metabolism (dimethylamine (DMA), dimethylglycine (DMG), choline) and the metabolism of dietary components (sucrose, glucose, tartrate) compared with those receiving the REF diet. Other metabolites excreted in lower amounts by the $\mathrm{C} 10$ group were those related to endogenous amino acid metabolism ( $\alpha$-keto-isocaproate ( $\alpha \mathrm{KIC}$ ), $\alpha$-keto$\beta$-methyl- $n$-valerate ( $\alpha \mathrm{KMV}$ ), $\beta$-hydroxy- $\beta$-methylbutyrate (HMB), 3-hydroxyisobutyrate, glycine) and metabolites arising from the gut microbial-host co-metabolism of amino acids (3-indoxylsulfate (3-IS), 4-cresyl sulfate (4-CS) and 4-cresyl glucuronide (4-CG)). Sebacate, 4-guanidinobutanoate, creatinine, allantoin and pseudouridine were also present in lower amounts in the urine samples of C10-fed animals compared with those in the REF group.

With regard to the $\mathrm{CF}$ diet, clear metabolic variation was observed in the urine samples of rats fed this diet compared with the samples of those fed the REF diet (Fig. 3(b) and 4; OPLS-DA model $\left.\mathrm{Q}^{2} \mathrm{Y}=0 \cdot 65 ; P=0 \cdot 001\right)$. Cocoa-derived metabolites such as NMNA, NMN, theobromine, xanthine, 1-MX, 3-MX, 7-MX and DMU were found in the urine samples of rats receiving the $\mathrm{CF}$ diet but not in that of those receiving the REF diet. Moreover, rats following the $\mathrm{CF}$ diet excreted higher 2-HIB, IAA, citrate, acetone, NMND, sucrose, glucose, acetate 
(a)

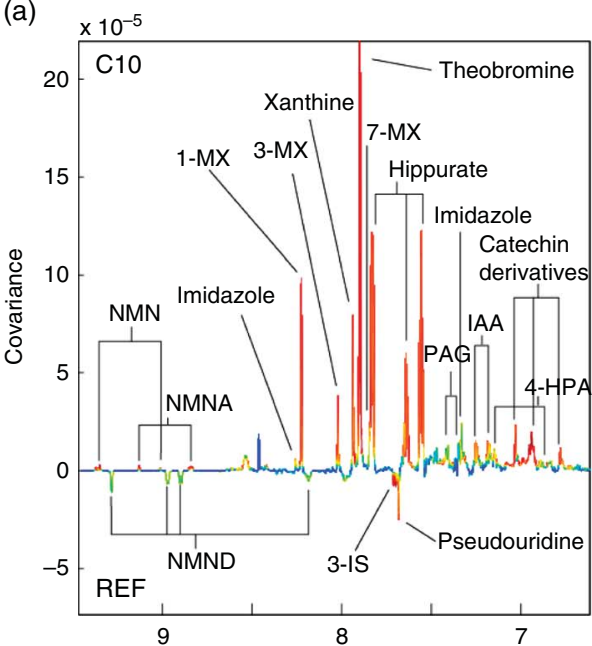

(b)

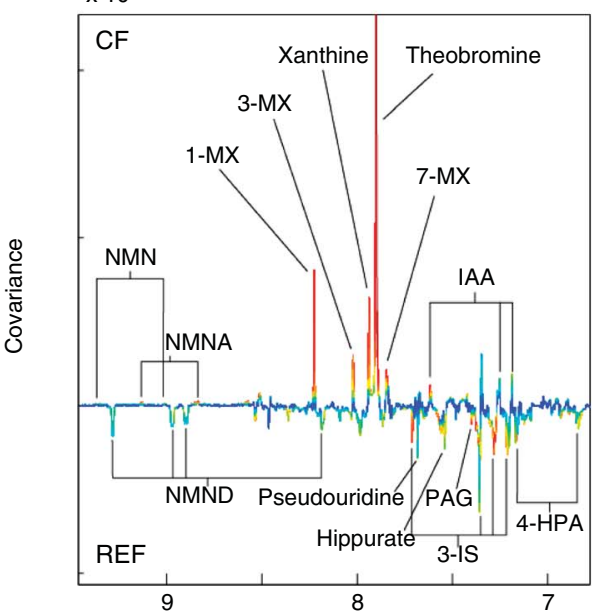

(c)

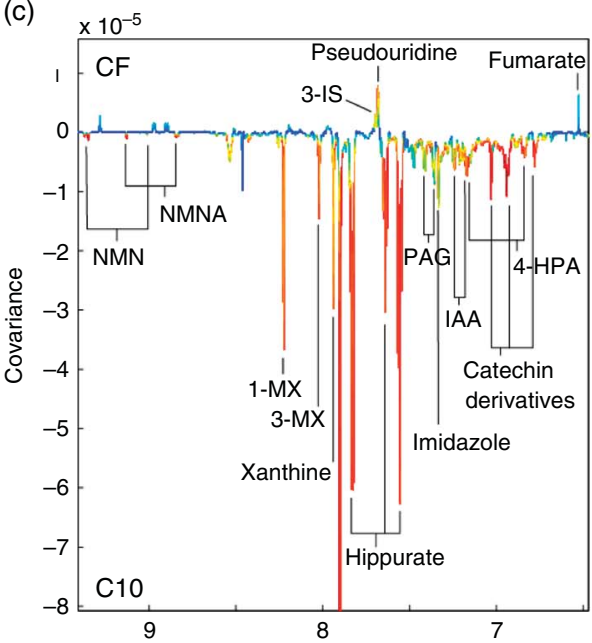

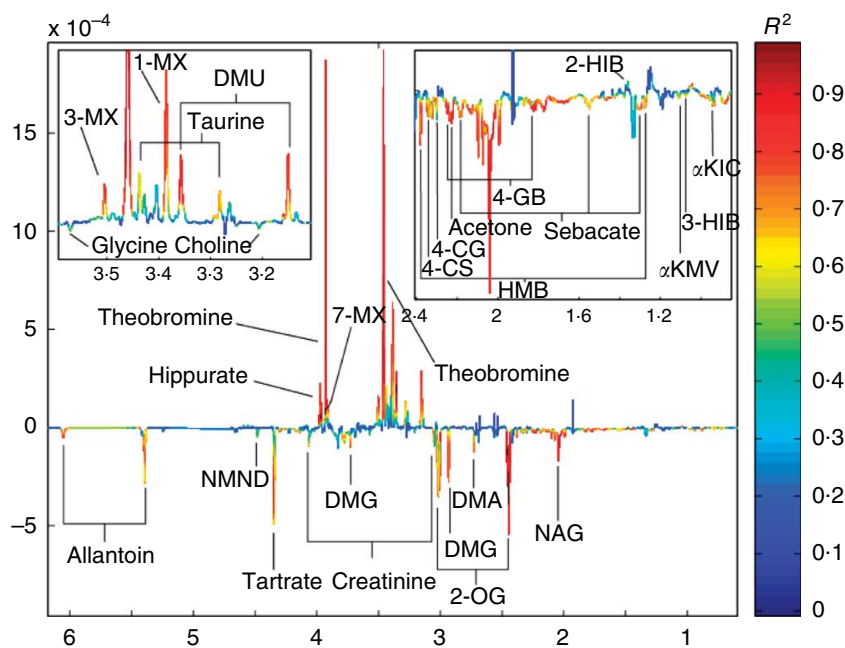
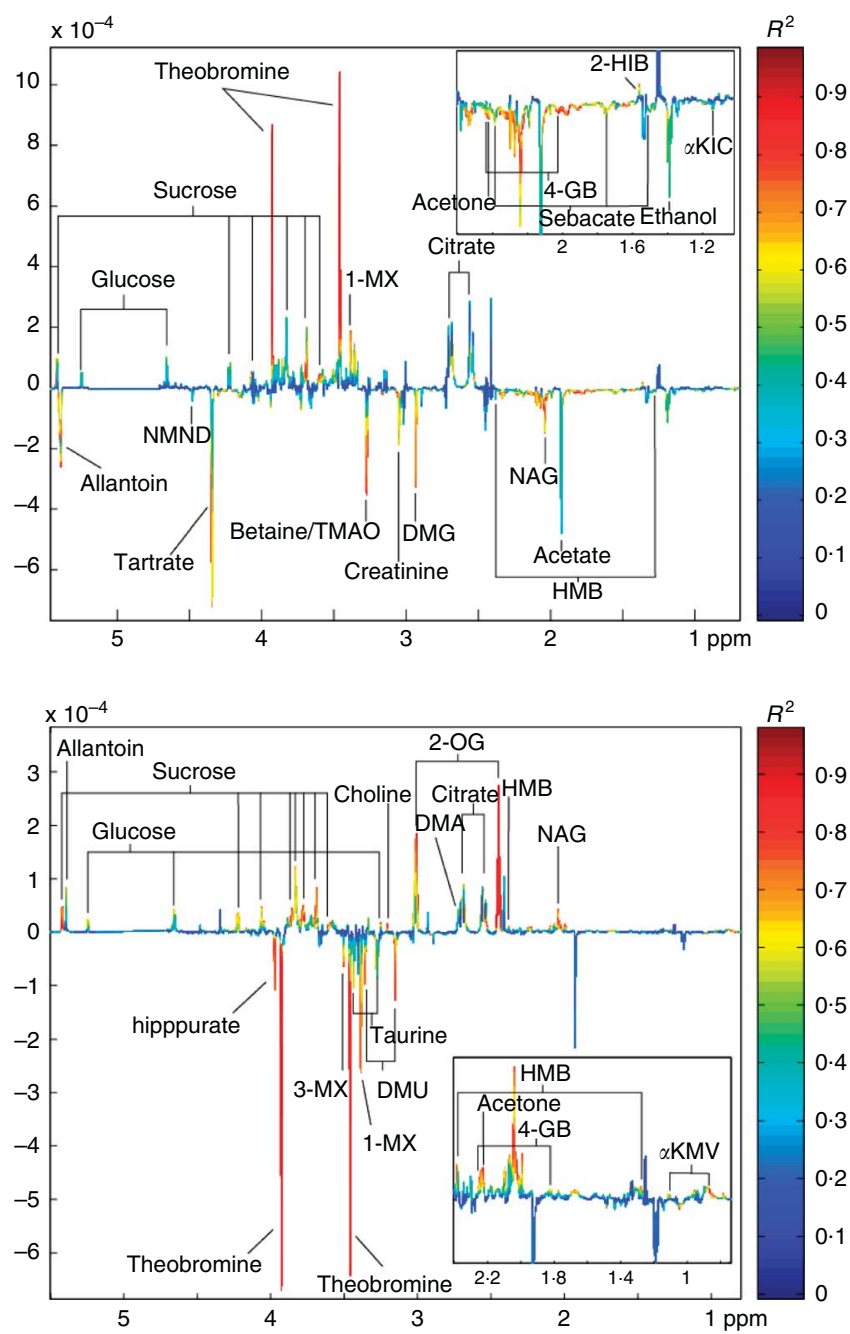

Fig. 3. Orthogonal projection to latent structures-discriminant analysis (OPLS-DA) comparing the urinary metabolic profiles of rats receiving different dietary regimens ( $n 8$ for the reference (REF) and the $10 \%$ cocoa (C10) groups; $n 5$ for the cocoa fibre (CF) group). Coefficient plots extracted from the OPLS-DA models comparing rats receiving (a) REF diet with C10 diet, (b) REF diet with CF diet and (c) C10 diet with CF diet. aKMV, $a$-keto- $\beta$-methyl- $n$-valerate; $a \mathrm{KIC}$, $a$-keto-isocaproate; 1 -MX, 1-methylxanthine; 2-HIB, 2-hydroxyisobutyrate; 2-OG, 2-oxoglutarate; 3-HIB, 3-hydroxyisobutyrate; 3-IS, 3-indoxyl-sulfate; 3-MX, 3-methylxanthine; 4-GB, 4-guanidinobutanoic acid; 4-CS, 4-cresyl sulfate; 4-CG, 4-cresyl glucuronide; 4-HPA, 4-hydroxypropionic acid; 7-MX, 7-methylxanthine; DMA, dimethylamine; DMG, dimethylglycine; DMU, dimethyluric acid; HMB, $\beta$-hydroxy- $\beta$-methylbutyrate; IAA, indole-3-acetic acid; NAG, $N$-acetylglycoprotein; NMN, nicotine mononucleotide; NMNA, $N$-methylnicotinic acid; NMND, $N$-methylnicotinamide; PAG, phenylacetylglycine; ppm, parts per million; TMAO, trimethylamine $N$-oxide. 


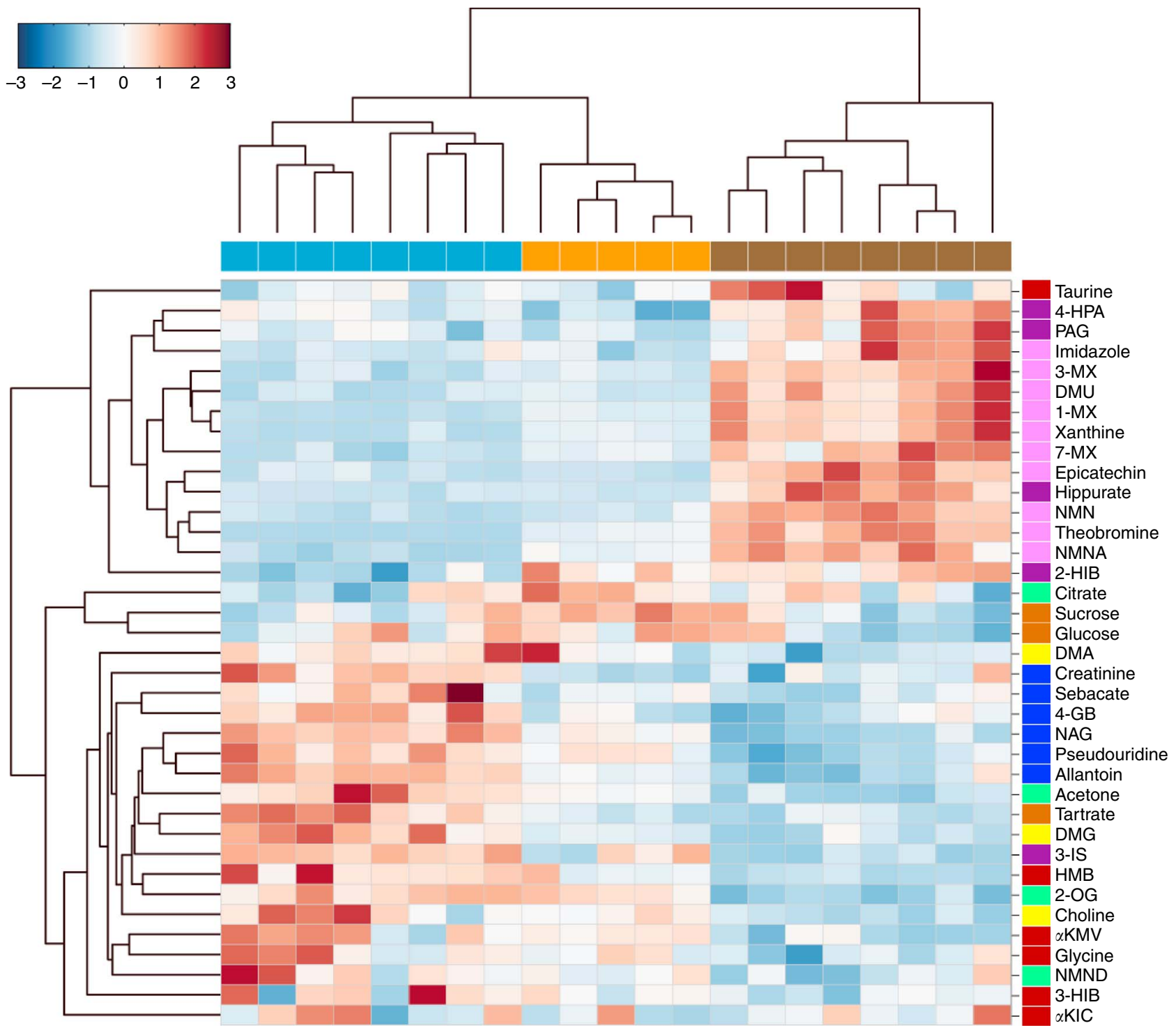

Fig. 4. Dendrogram and heatmap representation of unsupervised hierarchical clustering (HCA) of the metabonome for all rats. Each column corresponds to a single rat ( $n 8$ for the reference (REF) and the $10 \%$ cocoa (C10) groups; $n 5$ for the cocoa fibre (CF) group), and each row corresponds to a specific metabolite. Metabolites identified to contribute to the separation between diets through the orthogonal projection to latent structures-discriminant analysis models were used for sample clustering. Metabolite $z$-score transformation was performed on the levels of each metabolite across samples, with blue denoting a lower and red denoting a higher level compared with the mean. Metabolites and samples are clustered using correlation distance and average linkage and colour-coded by diet or pathway, respectively. HCA grouped the urinary metabolic profiles from the C10-fed animals together and distinct from the other studied animals. Profiles from animals receiving the CF diet clustered together and were separated from the REF diet. 4-HPA, 4-Hydroxypropionic acid; PAG, phenylacetylglycine; 3-MX, 3-methylxanthine; DMU, dimethyluric acid; 1-MX, 1-methylxanthine; 7-MX, 7-methylxanthine; NMN, nicotine mononucleotide; NMNA, N-methylnicotinic acid; 2-HIB, 2-hydroxyisobutyrate; DMA, dimethylamine; 4-GB, 4-guanidinobutanoic acid; NAG, $N$-acetylglycoprotein; DMG, dimethylglycine; 3-IS, 3-indoxyl-sulfate; HMB, $\beta$-hydroxy- $\beta$-methylbutyrate; 2-OG, 2-oxoglutarate; aKMV, $\alpha$-keto- $\beta$-methyl- $n$-valerate; NMND, $N$-methylnicotinamide; 3-HIB, 3-hydroxyisobutyrate; aKIC, $a$-keto-isocaproate. $\square$, REF; $\square$, CF; $\square, C 10 ; \square$, amino acid metabolism; $\square$, gut microbial metabolism; $\square$, cocoa derived; $\square$, energy metabolism; $\square$, choline metabolism; $\square$, miscellaneous; $\square$, dietary.

and tartrate than those following the REF diet, which are related to microbial, energetic or dietary metabolism. Lower amounts of 4-HPA, hippurate, 3-IS, PAG, $\alpha \mathrm{KI}$, DMG, sebacate, 4-guanidinobutanoate, ethanol, creatinine, allantoin and pseudouridine were also measured in the urine samples of the CF group compared with those in the REF group.

Finally, the OPLS-DA model contrasting the urinary metabolic phenotypes from rats receiving the $\mathrm{C} 10$ diet and those fed the CF diet (Fig. 3(c) and 4; $Q^{2} Y=0.89 ; P=0.001$ ) also showed some clear differences. As expected, rats consuming the
C10 diet excreted higher levels of cocoa- (NMNA, NMN), catechin- and methylxanthine- (theobromine, xanthine, 1-MX and 3-MX, imidazole, DMU) derivatives compared with that of the rats fed the $\mathrm{CF}$ diet. The $\mathrm{C} 10$ diet-fed animals also excreted higher amounts of 4-HPA, hippurate, PAG, IAA and taurine. However, compared with the CF group, the C10 diet-fed animals eliminated lower amounts of 3-IS, acetone, citrate, 2-OG, NMND, $\alpha \mathrm{KMV}$, HMB, DMA, choline, sucrose, glucose, sebacate, 4-guanidinobutanoate, allantoin, pseudouridine and fumarate. 
Correlations between urine metabolites and studied variables

The correlation analysis of the urine metabolic fingerprint with the effects of cocoa on metabolic hormones (ghrelin, GLP-1, glucagon, leptin), body weight, intestinal immunity (by means of intestinal IgA levels) and microbiota composition (reported previously $^{(17,26)}$ ) was also studied (Fig. 5). Globally, whereas most of the studied variables were inversely correlated with whole-cocoa metabolites, only the ghrelin concentration was positively correlated with the amount of these metabolites. In fact, the strongest correlations were found between ghrelin and NMNA, 1-MX, xanthine and theobromine. Plasma glucagon concentration correlated inversely with these metabolites derived from cocoa, whereas it had a positive correlation with choline and other metabolites. Body weight was inversely correlated with cocoa metabolites and positively correlated with those from the amino acid metabolism. Similarly, faecal IgA concentration showed an inverse correlation with cocoaderived metabolites, whereas it was positively correlated with choline metabolites and allantoin. Urinary metabolites were also correlated with gut microbial groups previously identified to change following both the $\mathrm{C} 10$ and $\mathrm{CF}$ intake ${ }^{(17)}$. An inverse correlation was found between the Streptococcus genus and the excretion of epicatechin (cocoa derived) and 4-HPA (gut microbial metabolism). In addition, a strong positive correlation was observed between the Firmicutes:Bacteroidetes ratio and choline-related metabolites, but an inverse correlation with metabolites derived from cocoa.

\section{Other correlations between studied variables}

The correlation analysis between the effects of cocoa on the metabolic hormones, body weight, intestinal immunity and microbiota composition and functionality ${ }^{(17)}$ has also been studied (Fig. 6). When samples from all the groups were considered together, body weight correlated positively with both plasma concentrations of glucagon and leptin, but negatively correlated with plasma ghrelin concentration. In addition, body weight showed a positive correlation with the faecal IgA content and the Streptococcus genus proportion, among others.

The metabolic hormones showed significant associations with the microbiota composition and functionality (Fig. 6). Particularly, glucagon concentration was positively correlated with the faecal counts of Bifidobacterium spp., whereas the ghrelin concentration correlated inversely with Bifidobacterium spp. proportion and the caecal butyric acid concentration. Finally, faecal IgA was positively associated with the faecal Streptococcus spp. counts and inversely with ghrelin concentration.

\section{Discussion}

We have previously reported that a $\mathrm{C} 10$ diet in rats results in a lower body weight increase, attenuates intestinal IgA secretion and modifies gut microbiota composition ${ }^{(13,17,26-29)}$. In the present study, we demonstrate that cocoa intake alters the metabolic hormones and results in a distinct urinary metabolic pattern. Moreover, correlations between urinary metabolites and those variables in which cocoa has an effect (i.e. body weight, metabolic hormones and intestinal immunity) were established, as well as between these modified variables. In addition, we have also correlated all these variables with the cocoa effects on microbiota composition and functionality from a previous study ${ }^{(17)}$. Interestingly, the effects of either whole cocoa or cocoa fibre on the metabolic hormones and urinary metabolic profiles were different, which reflects the role of nonfibre cocoa compounds as well as the interaction between fibre and non-fibre cocoa compounds in these effects. To strengthen this conclusion, further studies testing the contribution of cocoa polyphenols to the aforementioned effects related to the urinary metabolic profile and metabolic hormones should be carried out.

As expected, the urinary metabolic profiles of the C10 group animals showed the highest excretion of cocoa-derived metabolites, which is in line with previous controlled cocoa dietary intervention studies ${ }^{(30-32)}$. These metabolites include both the flavonoid and methylxanthine derivatives, some of them produced by the gut microbiota. This reinforces the fact that some of these cocoa compounds reach the colon intact, where they are metabolised by intestinal bacteria ${ }^{(6,8)}$. However, these metabolites were found in a lower amount in the urine samples of animals fed the cocoa fibre, confirming the lower concentrations of polyphenols and methylxanthines in the fibre powder used in the present study.

Rats fed a C10 diet excreted lower amounts of $\alpha \mathrm{KMV}$ and $\alpha \mathrm{KIC}$, both derived from the endogenous catabolism of the branched-chain amino acids (BCAA). In comparison with the CF diet, the amount of glucose, sucrose and tricarboxylic acid (TCA) cycle intermediates (citrate, 2-OG and fumarate) was lower in the C10 group, indicating that the TCA cycle was down-regulated after cocoa intake. These changes, together with the lower urinary excretion of acetone, suggest an alteration in energy metabolism through the modulation of ketogenesis, BCAA metabolism and the TCA cycle. This shift in energy strategy, together with the lower expression of genes involved in lipid metabolism ${ }^{(33)}$, may contribute to the reduction of body weight in $\mathrm{C} 10$-fed rats, reported here and in previous studies ${ }^{(23,26,28)}$. Changes in $\mathrm{NAD}^{+}$pathway metabolism further support an alteration in energy metabolism. Thus, $\mathrm{NAD}^{+}$ is required in TCA cycle and oxidative phosphorylation in the mitochondria to produce energy ${ }^{(34)}$. It can be synthesised from NMN and nicotinic acid, which were excreted in higher amounts by the $\mathrm{C} 10$ rats. On the other hand, the $\mathrm{NAD}^{+}$consuming enzymes all generate nicotinamide as a by-product, which can then be recycled to generate NMN by nicotinamide phosphoribosyltransferase. However, it can also be methylated by nicontiamide $N$-methyltransferase (NNMT) to NMND, which was excreted in lower amounts by rats following a C10 diet. Recently, it was suggested that inhibition of NNMT protected against diet-induced obesity by increasing energy expenditure $^{(35)}$. Therefore, the lower urinary excretion of NMND could also explain the lower body weight increase observed in the present study in C10 rats.

In addition, increased levels of NMND in urine samples were correlated with peroxisome proliferators ${ }^{(36)}$, which participate 


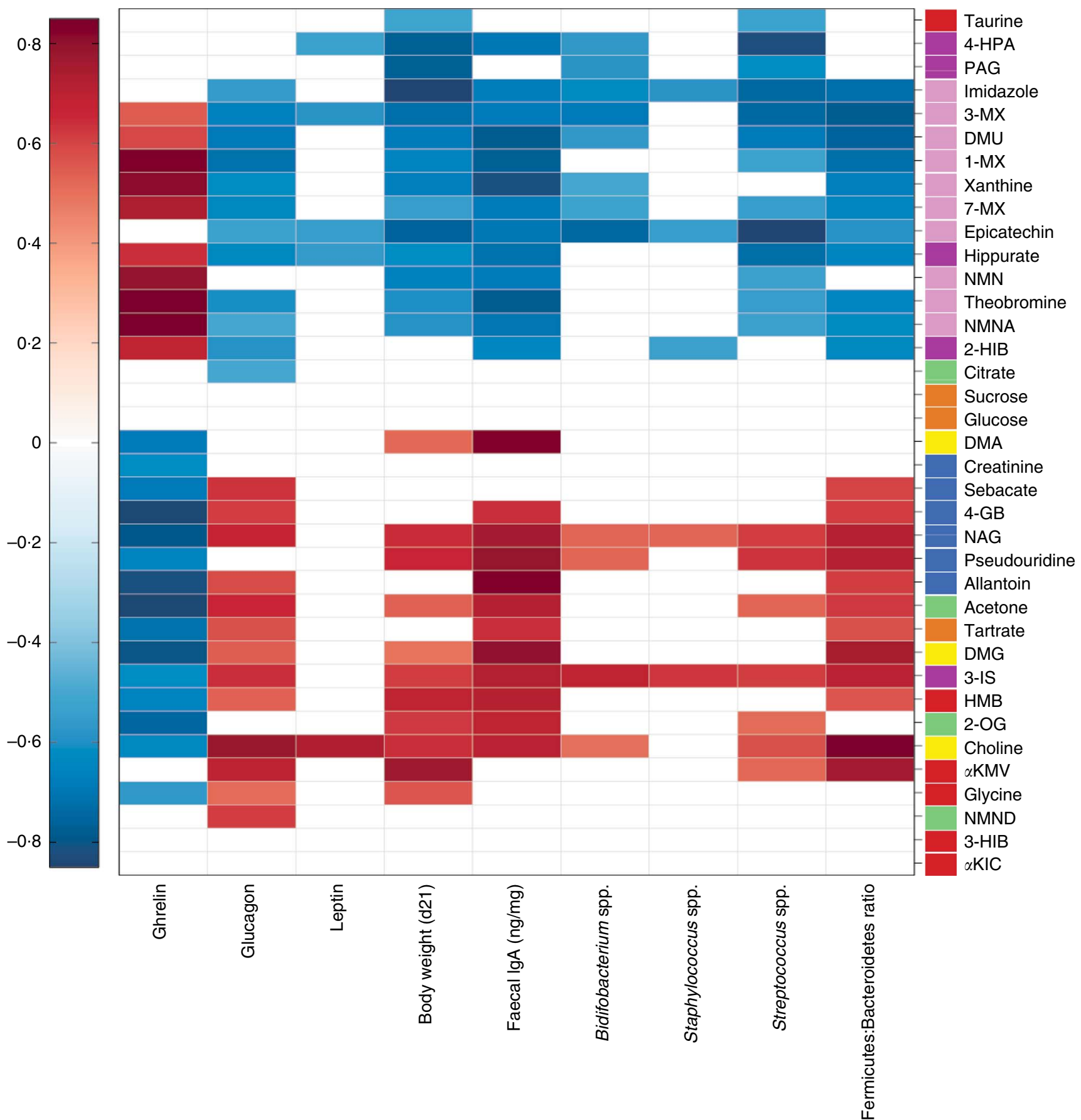

Fig. 5. Correlations between metabolites and responses. The intensity of the colours represents the degree of correlation, with red and blue indicating positive and negative correlations, respectively. Metabolites identified to contribute to the separation between diets through orthogonal projection to latent structures-discriminant analysis models were to obtain the correlations. The order of the metabolites is the same obtained in Fig. 4, where metabolites have been clustered based on an unsupervised hierarchical analysis using a correlation distance and average linkage and colour-coded by pathway. Only significant correlations after applying a Benjamini-Hochberg procedure for controlling for a false discovery rate of $5 \%$ are shown. Correlation coefficients were based on Spearman's correlation. 4-HPA, 4-hydroxypropionic acid; PAG, phenylacetylglycine; 3-MX, 3-methylxanthine; DMU, dimethyluric acid; 1-MX, 1-methylxanthine; 7-MX, 7-methylxanthine; NMN, nicotine mononucleotide; NMNA, $N$-methylnicotinic acid; 2-HIB, 2-hydroxyisobutyrate; DMA, dimethylamine; 4-GB, 4-guanidinobutanoic acid; NAG, $N$-acetylglycoprotein; DMG, dimethylglycine; 3-IS, 3-indoxyl-sulfate; HMB, $\quad \beta$-hydroxy- $\beta$-methylbutyrate; 2 -OG, 2-oxoglutarate; $a \mathrm{KMV}, \quad a$-keto- $\beta$-methyl- $n$-valerate; NMND, $N$-methylnicotinamide; 3-HIB, 3-hydroxyisobutyrate; $a \mathrm{KIC}$, a-keto-isocaproate. $\square$, amino acid metabolism; $\square$, gut microbial metabolism; $\square$, cocoa derived; $\square$, energy metabolism; $\square$, choline metabolism; $\square$, miscellaneous; $\square$, dietary.

in the generation of reactive oxygen species (ROS) ${ }^{(37)}$. Changes in $\mathrm{NAD}^{+}$metabolism have been associated with oxidative stress in aging and neurodegeneration ${ }^{(34)}$. Hence, the lower excretion of NMND after the C10 diet may reflect a reduction in oxidative stress. Consistent with this, rats maintained on a $\mathrm{C} 10$ diet excreted lower amounts of pseudouridine, which is the 


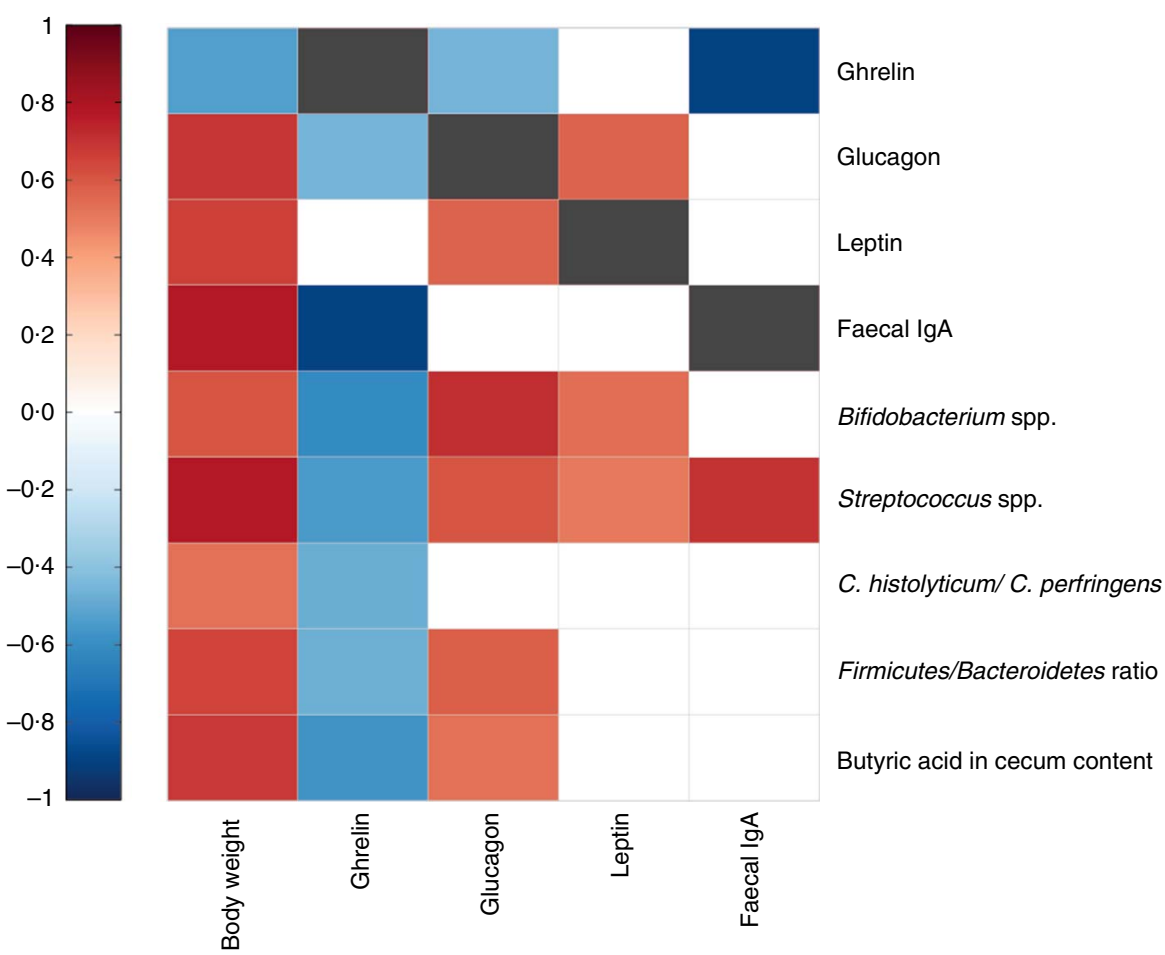

Fig. 6. Correlations between body weight, metabolic hormones, intestinal immunity and microbiota composition and functionality. The intensity of the colours represents the degree of correlation, with red and blue indicating positive and negative correlations, respectively. Only significant correlations after applying a Benjamini-Hochberg procedure for controlling for a false discovery rate of $5 \%$ are shown. Correlation coefficients were based on Spearman's correlation.

oxidised form of urinary nucleosides. Elevated levels of pseudouridine have been previously identified as indicators of liver damage induced by inflammation related to oxidative stress ${ }^{(38)}$. In addition, the gut microbiota produces potentially toxic substances derived from protein metabolism ${ }^{(39)}$, such as the uremic toxins 4-CS and 3-IS derived from tyrosine and tryptophan, respectively. Both uremic toxins have been shown to lead to changes in NAD metabolism, by increasing NADPH-oxidase activity, and to increase ROS production ${ }^{(40,41)}$. Hence, the lower urinary excretion of 4-CS, 4-CG and 3-IS by C10 rats strengthens the possible reduction in oxidative stress after cocoa consumption.

Microbial cocoa metabolism led to a higher excretion of 4-HPA, IAA, hippurate and PAG in the C10 group compared with that in both the CF and REF groups, which could arise from the differences in cocoa compounds between the diets. Cocoa is rich in monoamines, such as tyramine, tryptamine and 2-phenylethylamine, which can be degraded by gut bacteria to 4-HPA, IAA and PAG, respectively. These three metabolites can also be derived from the amino acid catabolism of tyrosine, tryptophan and phenylalanine by the gut microbiota, respectively. The gut microbiota can also degrade cocoa polyphenols to quinic acid and benzoic acid, which are then oxidised to hippurate and other phenol-related metabolites such as 4-HPA and PAG. Interestingly, NMND, 3-IS and pseudouridine were excreted in lower amounts after the C10 diet compared with that after the CF diet. This suggests a shift in the composition and functionality of the gut microbiota towards a more favourable and less oxidative profile. This further suggests that rather than its fibre content it is the other cocoa compounds that contribute to the beneficial effects of cocoa. An inverse correlation was observed between cocoa metabolites and body weight, the highest correlation being with imidazole, one of the smallest metabolites derived from methylxanthines. This clearly suggests that theobromine, a methylxanthine present in cocoa in a high concentration, and its metabolites contribute to the effects of cocoa on body weight. An inverse association was also found between body weight and the presence of metabolites derived from microbial metabolism, especially with PAG and 4-HPA. Regardless of other reported mechanisms ${ }^{(42-44)}$, the contribution of microbiota composition and its activity should be considered in the regulatory effect of cocoa on body weight. In fact, it has been shown that intake of the C10 diet decreased the abundance of the Staphylococcus and Streptococcus genera (from the Firmicutes phylum) and increased the Bacteroides spp. (Bacteroidetes phylum) reducing the Firmicutes:Bacteroidetes ratio ${ }^{(17,26)}$. This decreased ratio has been associated with lower body weight ${ }^{(45)}$. Consistently, an inverse correlation was also found between the counts of Streptococcus spp. and the excreted amounts of 4-HPA and epicatechin in urine.

The relationship between urinary metabolites and intestinal immunity has been established. Whereas the CF diet did not affect the IgA content, the C10 diet, in accordance with findings from previous studies ${ }^{(2,13,27)}$, resulted in a significantly lower faecal IgA concentration. In this sense, inverse correlations between the faecal IgA concentration and the amounts of cocoa epicatechin and methylxanthine derivatives and those from microbial metabolism were obtained. Overall, these results 
suggest that cocoa metabolism may lead to the formation of bioactive products that interact with the mechanisms involved in IgA secretion and/or its synthesis. Indeed, not only are the metabolites generated by the microbiota important but also the composition of the microbiota. Besides urinary metabolites and faecal IgA, metabolic hormones were determined after cocoa and the CF intakes. In line with previous studies ${ }^{(25)}$, the GLP-1 and glucagon, peptide hormones involved in the glucose metabolism, were reduced by the $\mathrm{C} 10$ diet, but only the former was also modified after the CF intake. However, only glucagon concentration correlated inversely with the cocoa metabolites. Regarding hormones involved in regulating food intake, leptin helps control the appetite and maintain a stable body weight ${ }^{(46)}$, whereas ghrelin stimulates appetite, increases fat body mass deposition and weight gain and influences glucose and lipid metabolism ${ }^{(47)}$. Although the $\mathrm{C} 10$ diet increased ghrelin concentration without changing leptin levels, it did not lead to changes in food intake. Therefore, it is unlikely that these regulating appetite hormones contribute to cocoa influence on body weight. In fact, ghrelin concentration was inversely associated with body weight. A two-way relationship between leptin and ghrelin and the gut microbiota has been suggested $^{(46,48)}$. Leptin stimulates mucin production in the intestine, which could affect the composition of the microbiota ${ }^{(49)}$. It has also been suggested that the microbiota may modulate these hormones through the physiological regulation of the levels and type of autoantibodies produced against them ${ }^{(50)}$. In this sense, and in line with previous studies ${ }^{(46)}$, we found that the proportions of Bifidobacterium and Streptococcus genera correlated positively with leptin and negatively with ghrelin. Moreover, it has been described that two of the main SCFA (butyrate and propionate) regulate gut hormone release ${ }^{(51)}$. In this regard, our study demonstrates that the ghrelin concentration was inversely correlated with the butyric acid concentration in caecum content. Therefore, although hormone effects on microbiota cannot be discarded, the cocoa modulatory effect on the microbiota composition and functionality might be partially responsible for the changes observed in both hormone concentrations.

In summary, through a metabonomic approach, we have described the urinary metabolites derived from cocoa and cocoa fibre intake that can be used as consumption markers in health conditions. Moreover, the lower excretion of certain host and microbial metabolites reflected a shift in the host energy and amino acid metabolism due to cocoa compounds, but not due to the CF diet. Metabolic associations were identified with body weight, intestinal IgA, the metabolic hormone profile and microbiota composition, extending our understanding of the mechanisms through which cocoa may impact on health. Such effects were not restricted solely to cocoa fibre, and the contribution of other cocoa compounds has been demonstrated. Further studies should be carried out to evaluate the precise contribution of cocoa methylxanthines and also to study these associations in the context of weight-altering stimulus.

\section{Acknowledgements}

The authors would like to thank the Genomic Services of the 'Centres Científics i Tecnològics' of the University of Barcelona and the NMR Laboratory of the Chemical Analysis Facility technicians from the University of Reading for their technical assistance. The authors also thank Idilia Foods S.L. and InnovaFood 2005 S.L. for providing the conventional cocoa and cocoa fibre powders and the inulin extract, respectively.

The present study was supported by a grant from the Spanish Ministry of Economy and Competitiveness (AGL2011-24279). M. M.-C. holds a fellowship from the Generalitat de Catalunya (grant no. 2014FI_B2 00048). None of the funders had a role in the design, analysis or writing of this article.

The authors' contributions were as follows: M. M.-C., À. F., F. J. P.-C. and M. C. conceived and designed the research; M. M.-C. and J. M.-P. carried out the metabolomics experiments, whereas M. M.-C. carried out the rest of the experiments; J. M.-P., J. R. S. and A. C. carried out the metabolomics data analysis and were involved in the interpretation of these data, whereas M. M.-C., F. J. P.-C. and M. C. carried out the Luminex and IgA data analysis and interpretation of the data; M. M.-C. and J. M.-P. contributed equally to the initial draft of the manuscript; A. C., J. R. S., À. F., F. J. P.-C. and M. C. contributed to the critical revision of the manuscript; F. J. P.-C. has primary responsibility for the final content. All authors have read and approved the final version of the manuscript for publication.

None of the authors has any conflicts of interest to declare.

\section{Supplementary material}

For supplementary material/s referred to in this article, please visit https://doi.org/10.1017/S0007114517000496

\section{References}

1. Hooper L, Kay C, Abdelhamid A, et al. (2012) Effects of chocolate, cocoa, and flavan-3-ols on cardiovascular health: a systematic review and meta-analysis of randomized trials. Am J Clin Nutr 95, 740-751.

2. Pérez-Cano FJ, Massot-Cladera M, Franch À, et al. (2013) The effects of cocoa on the immune system. Front Pharmacol 4, 71.

3. Castell M, Pérez-Cano FJ, Abril-Gil M, et al. (2014) Flavonoids on allergy. Curr Pharm Des 20, 973-987.

4. Lecumberri E, Goya L, Mateos R, et al. (2007) A diet rich in dietary fiber from cocoa improves lipid profile and reduces malondialdehyde in hypercholesterolemic rats. Nutrition $\mathbf{2 3}$, 3323-3341

5. Ramos S, Moulay L, Granado-Serrano AB, et al. (2008) Hypolipidemic effect in cholesterol-fed rats of a soluble fiberrich product obtained from cocoa husks. J Agric Food Chem 56, 6985-6993.

6. Monagas M, Urpi-Sarda M, Sánchez-Patán F, et al. (2010) Insights into the metabolism and microbial biotransformation of dietary flavan-3-ols and the bioactivity of their metabolites. Food Funct 1, 233-253.

7. Neilson AP \& Ferruzzi MG (2011) Influence of formulation and processing on absorption and metabolism of flavan-3-ols from tea and cocoa. Annu Rev Food Sci Technol 2, 125-151.

8. Selma MV, Espín JC \& Tomás-Barberán FA (2009) Interaction between phenolics and gut microbiota: role in human health. J Agric Food Chem 57, 6485-6501.

9. Hayek N (2013) Chocolate, gut microbiota, and human health. Front Pharmacol 4, 11. 
10. Lee HC, Jenner AM, Low CS, et al. (2006) Effect of tea phenolics and their aromatic fecal bacterial metabolites on intestinal microbiota. Res Microbiol 157, 876-884.

11. van Duynhoven J, Vaughan EE, van Dorsten F, et al. (2013) Interactions of black tea polyphenols with human gut microbiota: implications for gut and cardiovascular health. Am J Clin Nutr 98, 1631S-1641S.

12. Etxeberria U, Fernández-Quintela A, Milagro FI, et al. (2013) Impact of polyphenols and polyphenol-rich dietary sources on gut microbiota composition. J Agric Food Chem 61, 9517-9533.

13. Massot-Cladera M, Abril-Gil M, Torres S, et al. (2014) Impact of cocoa polyphenol extracts on the immune system and microbiota in two strains of young rats. Br J Nutr $\mathbf{1 1 2}$, 1944-1954.

14. Turnbaugh PJ, Ridaura VK, Faith JJ, et al. (2009) The effect of diet on the human gut microbiome: a metagenomic analysis in humanized gnotobiotic mice. Sci Transl Med 1, Gra14.

15. Wu GD, Chen J, Hoffmann C, et al. (2011) Linking long-term dietary patterns with gut microbial enterotypes. Science 334, 105-109.

16. Claesson MJ, Jeffery IB, Conde S, et al. (2012) Gut microbiota composition correlates with diet and health in the elderly. Nature 488, 178-184

17. Massot-Cladera M, Costabile A, Childs CE, et al. (2015) Prebiotic effects of cocoa fibre on rats.J Funct Foods 19, 341-352.

18. Holscher HD, Bauer LL, Gourineni V, et al. (2015) Agave inulin supplementation affects the fecal microbiota of healthy adults participating in a randomized, double-blind, placebocontrolled, crossover trial. J Nutr 145, 2025-2032.

19. Lecumberri E, Mateos R, Izquierdo-Pulido M, et al. (2007) Dietary fibre composition, antioxidant capacity and physicochemical properties of a fibre-rich product from cocoa (Theobroma cacao L.). Food Chem 104, 948-954.

20. Rios LY, Gonthier MP, Rémésy C, et al. (2003) Chocolate intake increases urinary excretion of polyphenol-derived phenolic acids in healthy human subjects. Am J Clin Nutr 77, 912-918.

21. Del Rio D, Rodriguez-Mateos A, Spencer JP, et al. (2013) Dietary (poly)phenolics in human health: structures, bioavailability, and evidence of protective effects against chronic diseases. Antioxid Redox Signal 18, 1818-1892.

22. Pérez-Berezo T, Franch À, Castellote C, et al. (2012) Mechanisms involved in down-regulation of intestinal IgA in rats by high cocoa intake. J Nutr Biochem 23, 838-844.

23. Ramiro-Puig E, Pérez-Cano FJ, Ramos-Romero S, et al. (2008) Intestinal immune system of young rats influenced by cocoaenriched diet. J Nutr Biochem 19, 555-565.

24. Massot-Cladera M, Franch À, Pérez-Cano FJ, et al. (2016) Cocoa and cocoa fibre differentially modulate IgA and IgM production at mucosal sites. BrJ Nutr 115, 1539-1546.

25. Abril-Gil M, Pérez-Cano FJ, Franch À, et al. (2016) Effect of a cocoa-enriched diet on immune response and anaphylaxis in a food allergy model in Brown Norway rats. J Nutr Biochem 27, 317-326.

26. Massot-Cladera M, Pérez-Berezo T, Franch À, et al. (2012) Cocoa modulatory effect on rat faecal microbiota and colonic crosstalk. Arch Biochem Biophys 527, 105-112.

27. Massot-Cladera M, Franch À, Castellote C, et al. (2013) Cocoa flavonoid-enriched diet modulates systemic and intestinal immunoglobulin synthesis in adult Lewis rats. Nutrients $\mathbf{5}$, 3272-3286

28. Pérez-Berezo T, Ramiro-Puig E, Pérez-Cano FJ, et al. (2009) Influence of a cocoa-enriched diet on specific immune response in ovalbumin-sensitized rats. Mol Nutr Food Res $\mathbf{5 3}$, 389-397.
29. Ramiro-Puig E, Pérez-Cano FJ, Ramírez-Santana C, et al. (2007) Spleen lymphocyte function modulated by a cocoaenriched diet. Clin Exp Immunol 149, 535-542.

30. Llorach R, Urpi-Sarda M, Tulipani S, et al. (2013) Metabolomic fingerprint in patients at high risk of cardiovascular disease by cocoa intervention. Mol Nutr Food Res 57, 962-973.

31. Garcia-Aloy M, Llorach R, Urpi-Sarda M, et al. (2015) A metabolomics-driven approach to predict cocoa product consumption by designing a multimetabolite biomarker model in free-living subjects from the PREDIMED study. Mol Nutr Food Res 59, 212-220.

32. Martínez-López S, Sarriá B, Gómez-Juaristi M, et al. (2014) Theobromine, caffeine, and theophylline metabolites in human plasma and urine after consumption of soluble cocoa products with different methylxanthine contents. Food Res Int 63, 446-455.

33. Massot-Cladera M, Franch À. Castell M, et al. (2016) Cocoa polyphenols and fiber modify colonic gene expression in rats. Eur J Nutr (epublication ahead of print version 2 June 2016).

34. Verdin E. (2015) NAD+ in aging, metabolism, and neurodegeneration. Science 350, 1208-1213.

35. Kraus D, Yang Q, Kong D, et al. (2014) Nicotinamide $\mathrm{N}$-methyltransferase knockdown protects against diet-induced obesity. Nature 508, 258-262.

36. Delaney J, Hodson MP, Thakkar H, et al. (2005) Tryptophan$\mathrm{NAD}+$ pathway metabolites as putative biomarkers and predictors of peroxisome proliferation. Arch Toxicol 79, 208-223.

37. Schrader M \& Fahimi HD (2006) Peroxisomes and oxidative stress. Biochim Biophys Acta 1763, 1755-1766.

38. Tamura S, Fujioka H, Nakano T, et al. (1988) Urinary pseudouridine as a biochemical marker in the diagnosis and monitoring of primary hepatocellular carcinoma. Am J Gastroenterol 83, 841-845.

39. Meyer TW \& Hostetter TH (2012) Uremic solutes from colon microbes. Kidney Int 81, 949-954.

40. Watanabe H, Miyamoto Y, Honda D, et al. (2013) p-Cresyl sulfate causes renal tubular cell damage by inducing oxidative stress by activation of NADPH oxidase. Kidney Int $\mathbf{8 3}$, 582-592.

41. Dou L, Jourde-Chiche N, Faure V, et al. (2007) The uremic solute indoxyl sulfate induces oxidative stress in endothelial cells. J Thromb Haemost 5, 1302-1308.

42. Gu Y, Hurst WJ, Stuart DA, et al. (2011) Inhibition of key digestive enzymes by cocoa extracts and procyanidins. J Agric Food Chem 59, 5305-5311.

43. Farhat G, Drummond S, Fyfe L, et al. (2014) Dark chocolate: an obesity paradox or a culprit for weight gain? Phyther Res 28, 791-797.

44. Rabadan-Chávez G, Quevedo-Corona L, Garcia AM, et al. (2016) Cocoa powder, cocoa extract and epicatechin attenuate hypercaloric diet-induced obesity through enhanced $\beta$-oxidation and energy expenditure in white adipose tissue. J Funct Foods 20, 54-67.

45. Sanz Y, Rastmanesh R \& Agostonic C (2013) Understanding the role of gut microbes and probiotics in obesity: how far are we? Pharmacol Res 69, 144-155.

46. Queipo-Ortuño MI, Seoane LM, Murri M, et al. (2013) Gut microbiota composition in male rat models under different nutritional status and physical activity and its association with serum leptin and ghrelin levels. PLOS ONE 8, e65465.

47. De Vriese C \& Delporte C (2008) Ghrelin: a new peptide regulating growth hormone release and food intake. Int $J$ Biochem Cell Biol 40, 1420-1424.

48. Ravussin Y, Koren O, Spor A, et al. (2012) Responses of gut microbiota to diet composition and weight loss in lean and obese mice. Obesity 20, 738-747. 
49. El Homsi M, Ducroc R, Claustre J, et al. (2007) Leptin modulates the expression of secreted and membrane-associated mucins in colonic epithelial cells by targeting PKC, PI3K, and MAPK pathways. Am J Physiol Gastrointest Liver Physiol 293, 365G-373G.

50. Fetissov SO, Hamze Sinno M, Coëffier M, et al. (2008) Autoantibodies against appetite-regulating peptide hormones and neuropeptides: putative modulation by gut microflora. Nutrition 24, 348-359.

51. Lin HV, Frassetto A, Kowalik EJ, et al. (2012) Butyrate and propionate protect against diet-induced obesity and regulate gut hormones via free fatty acid receptor 3-independent mechanisms. PLOS ONE 7, e35240. 